
\title{
28 Research Square \\ Expression Status and Prognostic Value of m6A RNA Methylation Regulators in Lung Adenocarcinoma
}

\section{Rui Wang}

Anhui Provincial Hospital Affiliated to Anhui Medical University

\section{Zian Feng}

The First Affiliated Hospital of USTC: Anhui Provincial Hospital

Jie Hu

The First Affiliated Hospital of USTC: Anhui Provincial Hospital

\section{Xiaodong He}

Anhui Provincial Center for Clinical Laboratories

\section{Zuojun Shen ( $\boldsymbol{\nabla}$ zuojunshen@ustc.edu.cn )}

The First Affiliated Hospital of USTC, Division of Life Sciences and Medicine, University of Science and Technology of China https://orcid.org/0000-0001-7052-5652

\section{Primary research}

Keywords: m6A methylation, lung adenocarcinoma, prognostic signature, survival analysis

Posted Date: December 4th, 2020

DOl: https://doi.org/10.21203/rs.3.rs-120253/v1

License: (c) (i) This work is licensed under a Creative Commons Attribution 4.0 International License. Read Full License 


\section{Abstract}

Background: $\mathrm{N}^{6}$-methyladenosine $\left(\mathrm{m}^{6} \mathrm{~A}\right) \mathrm{RNA}$ modification is the most abundant modification method in mRNA, and it plays an important role in the occurrence and development of many cancers. However, data on the role of $\mathrm{m}^{6} \mathrm{~A}$ RNA methylation regulators in lung adenocarcinoma (LUAD) are still lacking. This paper mainly discusses the role of $\mathrm{m}^{6} \mathrm{~A}$ RNA methylation regulators in LUAD, to identify novel prognostic biomarkers.

Methods: The gene expression data of $19 \mathrm{~m}^{6} \mathrm{~A}$ methylation regulator in LUAD patients and its relevant clinical parameters were extracted from The Cancer Genome Atlas (TCGA) database. The least absolute shrinkage and selection operator (LASSO) Cox regression algorithm were performed to construct a risk signature and evaluated its prognostic prediction efficiency by using the receiver operating characteristic (ROC) curve. The risk score of each patient was calculated according to the risk signature, and LUAD patients were divided into high-risk group and low-risk group. Kaplan-Meier survival analysis and Cox regression analysis were used to identify the independent prognostic significance of risk signature. Finally, Gene Ontology (GO), Kyoto Encyclopedia of Genes and Genomes (KEGG), and Gene Set Enrichment Analysis (GSEA) were used to explore the differential signaling pathways and cellular processes between the two groups.

Results: The expression of $15 \mathrm{~m}^{6} \mathrm{~A}$ RNA methylation regulators in LUAD tissues was significantly different than that in normal tissues. YTHDF3, YTHDF2, KIAA1429, HNRNPA2B1, RBM15, METTL3, HNRNPC, YTHDF1, IGF2BP2, IGF2BP3, IGF2BP1 were significantly up-regulated in LUAD, and the expressions of FTO, ZC3H13, WTAP, and METL14 were significantly down-regulated. We selected IGF2BP1, HNRNPC, and HNRNPA2B1 to construct the risk signature. ROC curve indicated the area under the curve (AUC) was 0.659 , which means the risk signature had a good prediction efficiency. The results of Kaplan-Meier survival analysis and Cox regression analysis showed that the risk score can be used as an independent prognostic factor for LUAD.

Conclusions: The $\mathrm{m}^{6} \mathrm{~A}$ RNA methylation regulators IGF2BP1, HNRNPC, and HNRNPA2B1 have a significant correlation with the clinicopathological characteristics of LUAD, which may be a promising prognostic feature and clinical treatment target.

\section{Background}

Lung cancer is the leading cause of cancer-related deaths worldwide. (1) There are many risk factors for lung cancer, with smoking, environmental, and occupational exposure as the most common risk factors. (2) In the past few decades, medical technology has made great progress, but the treatment effect for lung cancer patients is not ideal. The 5-year survival rate for lung cancer is reported to be $19 \%$, one of the lowest five-year survival rates, while adenocarcinoma, the most common histological subtype of lung cancer, is more aggressive and has a poorer prognosis. (3-5) To relieve the current clinical treatment 
pressure and improve the prognosis of patients, it is necessary to find reliable prognostic markers to optimize the treatment regimen for lung adenocarcinoma (LUAD).

N6-methyladenosine $\left(\mathrm{m}^{6} \mathrm{~A}\right)$ is a methylated modification of RNA molecules that was first discovered in 1974. (6) As of the end of 2017, more than 150 post-transcriptional modifications have been identified in all organisms, and $\mathrm{m}^{6} \mathrm{~A}$ is the most common internal mRNA modification found in eukaryotes and plays a key role in a variety of basic biological processes such as cell differentiation, tissue development, and tumorigenesis. (7-10) In mammals, about $0.1-0.4 \%$ of adenosine in isolated RNA is $\mathrm{m}^{6} \mathrm{~A}$ modified, accounting for about $50 \%$ of the total methylated ribonucleotide. (11) Dominissini et al. (12) used a new method of antibi-mediated capture and massively parallel sequencing, $m^{6} \mathrm{~A}-\mathrm{seq}$, to find that $\mathrm{m}^{6} \mathrm{~A}$ is clustered in the termination codon, the $3^{\prime}$ untranslated region ( $3^{\prime}$ UTR), and the internal exon. $\mathrm{m}^{6} \mathrm{~A}$ methylation is a dynamic reversible process catalyzed by three types of proteases: Writers (Methyltransferase complex, including METTL3/14/16, WTAP, IC3H13, ZC3H13, RBM15/15B, and KIAA1429), Erasers (Demethylases, including FTO and ALKBH5) and Readers (including YTHDF1/2/3, IGF2BP1/2/3, YTHDC1/2, HNRNPC, HNRNPG, and HNRNPA2B1). $(11,13,14)$ Writers mediate the methylation modification process of RNA to "write" the methylation modification to RNA, and Readers are responsible for "reading" the information of RNA methylation modification, and participate in downstream RNA translation and degradation processes, and then rely on Erasers Mediating the process of RNA demethylation modification can "erased" the RNA methylation modification signal, thereby making the $\mathrm{m}^{6} \mathrm{~A}$ modification process dynamic and reversible. (15)

At present, many studies have shown that $\mathrm{m}^{6} \mathrm{~A}$ methylation regulators are closely related to the occurrence and development of tumors. For example, Taketo et al. (16) indicated that METL3, as an $\mathrm{m}^{6} \mathrm{~A}$ regulator, is up-regulated in patients with pancreatic cancer and is an effective target in the treatment of the patients. Maetal. et al. (17) found that down-regulation of METTL14 expression is a poor prognostic factor for hepatocellular carcinoma and is closely related to tumor metastasis. However, there is still insufficient information about the role of $\mathrm{m}^{6} \mathrm{~A}$ RNA methylation regulators in LUAD. Therefore, in this study, RNA sequencing data were obtained from The Cancer Genome Atlas (TCGA), and the expression data of $19 \mathrm{~m}^{6} \mathrm{~A}$ methylation regulators in 535 LUAD patients and 59 normal people were systematically analyzed, as well as their association with clinicopathological characteristics. We used least absolute shrinkage and selection operator (LASSO) Cox regression algorithm to analyze $19 \mathrm{~m}^{6} \mathrm{~A}$ methylation regulators, and selected IGF2BP1, HNRNPC, and HNRNPA2B1 to construct the minimum standard risk signature, and the Kaplan-Meier survival analysis, univariate, and multivariate Cox regression analysis were used to identify the predictive effect of the risk signature on LUAD patient prognosis. Gene Set Enrichment Analysis (GSEA), Gene Ontology (GO), and Kyoto Encyclopedia of Genes and Genomes (KEGG) analysis were used for further functional annotation.

\section{Methods}

\subsection{Data Acquisition}


All data in this study were downloaded from the TCGA (https://cancergenome.nih.gov/) database, including gene expression data (RNA-seq) and corresponding clinical information of 535 LUAD patients and 59 normal people. If any parameter value was missing, the entire patient data will be excluded from the analysis. After screening, 479 clinical data of LUAD patients were retained. (Additional file 1: Table S1)

\subsection{Selection of $\mathrm{m}^{6} \mathrm{~A}$ methylation regulators and analysis of their differential expression}

The TCGA database provides the expression data of $19 \mathrm{~m}^{6} \mathrm{~A}$ methylation regulators, including YTHDF3, YTHDF2, YTHDF1, KIAA1429, HNRNPA2B1, RBM15, METTL3, HNRNPC, IGF2BP2, IGF2BP3, IGF2BP1, FTO, ZC3H13, WTAP, METTL14, ALKBH3, ALKBH5, YTHDC1, and YTHDC2. In order to identify the expression of $\mathrm{m}^{6} \mathrm{~A}$ RNA methylation regulators in LUAD, the limma package (18) was used to analyze the expression of $19 \mathrm{~m}^{6} \mathrm{~A}$ RNA methylation regulators in of 479 LUAD tissues and 59 normal tissues, and the expression levels of $19 \mathrm{~m}^{6} \mathrm{~A}$ RNA methylation regulators in LUAD tissues with different clinical characteristics were compared, and the expression levels were evaluated by t-test. The result used the pheatmap package to generate heat map and vioplot to visualize.

\subsection{Correlation Analysis Of Ma Methylation Regulators}

In order to further study the correlation between $\mathrm{m}^{6} \mathrm{~A}$ methylation regulators, the co-expression correlation analysis was carried out, and the results were visualized by the "corrlot" package. We performed univariate Cox regression analysis on the expression of $19 \mathrm{~m}^{6} \mathrm{~A}$ RNA methylation regulators in 479 LUAD tumor tissues, and genes with $\mathrm{P}<0.05$ were considered to be significantly associated with the survival of LUAD patients.

\subsection{Construction And Verification Of Risk Signature}

To verify the prognostic effect of $\mathrm{m}^{6} \mathrm{~A}$ RNA methylation regulators in LUAD patients, we performed Lasso Cox regression analysis $(19,20)$ on $15 \mathrm{~m}^{6} \mathrm{~A}$ RNA methylation regulators significantly related to patient survival, and screened $3 \mathrm{~m}^{6} \mathrm{~A}$ RNA methylation regulators (IGF2BP1, HNRNPC, and HNRNPA2B1) to construct a minimum standard risk signature. (Additional file 2: Fig. S1a-b) The obtained coefficients (IGF2BP1 coefficient $=0.0352$, HNRNPC coefficient $=0.0046$, and HNRNPA2B1 coefficient $=0.0006$ ) were used to calculate the risk score of the TCGA dataset. The risk score calculation formula $\left(\right.$ Coef $_{i}$ means coefficient and $\operatorname{Exp}_{\mathrm{i}}$ means the expression value of each selected gene):

Risk score $=\sum_{i=1}^{n} \operatorname{Coef}_{\mathrm{i}} * \operatorname{Exp}_{\mathrm{i}}$ 
And patients were classified into low-risk and high-risk group according to the median of risk scores, and performed principal component analysis (PCA) on the grouping results. PCA was performed by the Limma package, and the results were visualized by the ggplot2 package. Survival package (21) was used to compare the overall survival (OS) rate of the high-risk and low-risk groups by the Kaplan-Meier method. Then we constructed a receiver operating characteristic (ROC) curve (22) to evaluated the prediction efficiency of the risk signature.

\subsection{Analysis Of Prognostic Ability Of The Three-gene Signature}

The "pheatmap" package was used to generate heat maps to visually analyze the expression differences of the three genes in the high-risk and low-risk groups, as well as the expression differences of the three genes in LUAD patients with different clinicopathological characteristics. Univariate, and multivariate independent prognostic analysis of the risk score were performed to identify the prognostic value of the risk signature. GSEA, were used to annotate the differential signaling pathways and cellular processes between the two groups. GO (23) enrichment, and KEGG (24) pathway analysis were used to analyze the differentially expressed genes (DEGs) between the high-risk group and the low-risk group. When the $P$ value was less than 0.05 , the enrichment pathway was considered to be statistically significant.

\subsection{Statistical Analysis}

One-way analysis of variance was used to compare the expression of $\mathrm{m}^{6} \mathrm{~A}$ RNA methylation regulators in tumor tissues of TCGA lung adenocarcinoma patients. The relationship between $\mathrm{m}^{6} \mathrm{~A}$ RNA methylation regulator and clinicopathological characteristics of LUAD patients was analyzed by t-test. OS was defined as the time interval from the date of diagnosis to the date of death. The Kaplan-Meier method was used for OS analysis to conduct a bilateral log-rank test. All statistical analyses were performed using $\mathrm{R}$ software (version 3.6.2). $P<0.05$ was considered statistically significant.

\section{Result}

\subsection{Expression of $\mathrm{m}^{6} \mathrm{~A}$ RNA methylation regulators in LUAD}

We compared the expression levels of $19 \mathrm{~m}^{6} \mathrm{~A}$ RNA methylation regulators in of LUAD tissues and 59 normal tissues extracted from the TCGA database. The results as shown in Fig. 1a and b, we found that there was a significant difference in the expression level of YTHDF3, YTHDF2, YTHDF1, KIAA1429, HNRNPA2B1, RBM15, METTL3, HNRNPC, IGF2BP2, IGF2BP3, IGF2BP1, FTO, ZC3H13, WTAP, METTL14 between LUAD carcinoma and normal tissues. Among these regulators, the expression level of YTHDF3, YTHDF2, KIAA1429, HNRNPA2B1, RBM15, METTL3, HNRNPC, YTHDF1, IGF2BP2, IGF2BP3, IGF2BP1 in 
LUAD was significantly higher than that in normal tissues, while FTO, ZC3H13, WTAP, METTL14 was lower than that in normal tissues.

\subsection{Correlation among the $19 \mathrm{~m}^{6} \mathrm{~A}$ RNA methylation regulators in LUAD}

To further understand the correlation among the $19 \mathrm{~m}^{6} \mathrm{~A}$ RNA methylation regulators in LUAD, we analyzed the correlation of $19 \mathrm{~m}^{6} \mathrm{~A}$ RNA methylation regulators. The results are shown in Fig. 2a, there are obvious correlations among the $19 \mathrm{~m}^{6} \mathrm{~A}$ RNA methylation regulators, and most of them are positive correlations. Among them, YTHDC2 and RBM15, YTHDC1 and METTL14, YTHDC2, and METL14 have the strongest positive correlation.

We performed univariate Cox regression analysis on $19 \mathrm{~m}^{6} \mathrm{~A}$ methylation regulators to identify the regulators in the LUAD dataset that were associated with the survival of LUAD patients. The results as shown in Fig. 2b, the expression of IGF2BP1 $(\mathrm{HR}=1.054, \mathrm{Cl}=1.028-1.081)$, IGF2BP2 $(\mathrm{HR}=1.025, \mathrm{Cl}=$ $1.007-1.043)$, IGF2BP3 $(\mathrm{HR}=1.065, \mathrm{Cl}=1.024-1.107), \mathrm{HNRNPC}(\mathrm{HR}=1.015, \mathrm{Cl}=1.005-1.025)$, RBM15 $(\mathrm{HR}=1.125, \mathrm{Cl}=1.014-1.249)$, HNRNPA2B1 $(\mathrm{HR}=1.007, \mathrm{Cl}=1.002-1.012)$, and KIAA1429 (HR $=1.064, \mathrm{Cl}=1.008-1.124)$ were significantly associated with the survival of LUAD patients, while IGF2BP1 $(P<0.001)$ was most related to the survival of LUAD patients.

\subsection{Construction Of The Ma-related Risk Signature}

To explore the prognostic value of the three-gene risk signature, we divided the LUAD patients obtained from TCGA into a low-risk group and a high-risk group based on the median risk score. As shown in Fig. 3a, as the risk score increases, the number of deaths in the high-risk group is significantly higher than that in the low-risk group. We also performed PCA on the risk signature to compare the differences between the two groups. The results showed that the distribution directions of the two groups were different and there was a clear boundary, suggesting that the risk signature can divide LUAD patients into two groups. (Fig. 3b) Then we constructed a Kaplan-Meier survival curve to analyze the OS rate of the two groups of patients. The results are shown in Fig. 3c, there is a significant difference in the OS rate between the high-risk group and the low-risk group $(P=1.257 \mathrm{e}-04)$. The OS rate of LUAD patients in the low-risk group is significantly higher than that of the LUAD patients in the high-risk group. In the followup, the ROC curve was established to evaluate the efficiency of the risk signature for predicting the 5-year survival rate of LUAD patients. As shown in Fig. $3 d$, the AUC was 0.659 , indicating that the risk signature has a good predictive efficiency on the 5-year survival rate of LUAD patients.

\subsection{Prognostic Analysis Of The Ma-related Risk Signature}

After dividing the high-risk group and the low-risk group according to the median risk score, we further compared the clinicopathological characteristics and the expression of three genes between the two groups of patients. The heat map in Fig. 4a showed that there were significant differences at T, stage, and 
status between the high-risk group and the low-risk group, and the expression of IGF2BP1, HNRNPC, and HNRNPA2B1 in the high-risk group was up-regulated, while in the low-risk group was down-regulated.

To verify whether the risk signature can be used as an independent prognostic indicator of LUAD, we conducted univariate and multivariate independent prognostic analysis on the risk score. As shown in Fig. $4 \mathrm{~b}$ and $\mathrm{c}$, the results of univariate Cox independent prognostic analysis showed that risk score

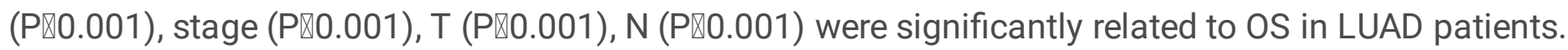
The results of multivariate Cox independent prognosis analysis showed that risk score $(P<0.001)$, stage $(P=0.002), N(P=0.031)$, which means the risk signature can be used as an independent prognostic factor for LUAD.

\subsection{Functional Enrichment Analysis}

We used GSEA to analyze the signal pathways of different prognosis between the high-risk group and the low-risk group. There are dramatic differences in the expression of genes involved in some pathways between the two groups. For example, genes involved in the cell cycle, genetic material metabolism, gene replication, and translation process are significantly up-regulated in the high-risk group, (Fig. 5) and these pathways are obviously related to the occurrence and development of cancer. After GESA analysis, we screened out a total of 378 DEGs (Log FC > 1, P < 0.05) between the high-risk and low-risk groups. To explore the differences in signal pathways and cellular processes between the two groups, we performed GO enrichment and KEGG pathway analysis on DEGs. The GO analysis results showed that DEGs were mainly enriched in tubulin binding, microtubule binding, and ATPase activity, while the KEGG analysis results showed that DEGs are mainly enriched in the cell cycle. (Fig. 6a and b)

\section{Discussion}

Lung adenocarcinoma is the most common subtype of non-small-cell lung cancer, accounting for almost $50 \%$ of all lung cancers, and its incidence is increasing year by year and overall survival is lower than that of most cancers. (5) Traditional treatment methods include surgery, radiotherapy, and chemotherapy. (25) The choice of treatment depends on the type of cancer (small cell or non-small cell), stage of development, and genetic characteristics. (26) If it can be detected at an early stage, surgical removal of non-small cell lung cancer can provide a good prognosis. However, more than $70 \%$ of patients with nonsmall cell lung cancer are diagnosed with advanced or metastatic disease. (27) Therefore, there is an urgent need to explore potential prognostic biomarkers and promising targets.

As the most common modification in human mRNA, ${ }^{6} \mathrm{~A}$ methylation modification is involved in regulating mRNA processing, translation, and stability. (28) Many studies have confirmed that $\mathrm{m}^{6} \mathrm{~A}$ RNA modification is related to tumor proliferation, differentiation, tumorigenesis, invasion, and metastasis. (17, $29,30)$ However, the role of $\mathrm{m}^{6} \mathrm{~A}$ methylation regulators in the occurrence and development of LUAD 
needs to be further clarified. Therefore, in this study, we explored the role of $\mathrm{m}^{6} \mathrm{~A}$ RNA methylation regulators in LUAD.

Different $\mathrm{m}^{6} \mathrm{~A}$ RNA methylation regulators have different effects on the same cancer, which may be related to their differential expression in different cancers. For example, as an "eraser" in the process of $\mathrm{m}^{6} \mathrm{~A}$ RNA methylation, FTO is highly expressed in breast, liver, and gastric cancer tissues compared to normal tissues, and is associated with poor prognosis, while FTO expression in bladder cancer tissues is lower than that in normal tissues. (31-34) Interestingly, in this study, we analyzed the data of 479 LUAD patients extracted from the TCGA database and showed that the $19 \mathrm{~m}^{6} \mathrm{~A}$ RNA methylation regulators we studied had abnormal expression in LUAD patients. Among them, the expression of FTO in cancer tissues of LUAD patients is significantly lower than that in normal tissues, which further validates the above viewpoint.

Insulin-like growth factor 2 mRNA-binding proteins (IGF2BPs, IGF2BP1/2/3), as a newly discovered $\mathrm{m}^{6} \mathrm{~A}$ regulator in recent years, promotes the stability and storage of its target mRNA (such as MYC) in an $m^{6} A-$ dependent manner, and play a carcinogenic effect in cancer. $(35,36)$ Interestingly, we performed univariate Cox regression analysis on $19 \mathrm{~m}^{6} \mathrm{~A}$ regulatory factors and found that IGF2BPs were significantly related to the survival of LUAD patients, and were significantly highly expressed in cancer tissues of LUAD patients. Therefore, we believe that the high expression of IGF2BPs may be one of the factors leading to the occurrence and development of LUAD in patients.

Next, we selected three $\mathrm{m}^{6} \mathrm{~A}$ RNA methylation regulators (IGF2BP1, HNRNPC, and HNRNPA2B1) to construct a minimum standard risk signature, which had a good predictive effect on the prognosis of LUAD patients. The higher the signature-based risk score, the worse the prognosis. At the same time, we divided 479 LUAD patients into high-risk and low-risk groups based on the median risk score, and verified the results with PCA and showed that the results of grouping according to the median risk score have a clear boundary, and Kaplan-Meier survival analysis results show that the signature can significantly distinguish LUAD patients with different OS, and also has a good predictive efficiency on the prognosis of LUAD patients (AUC $=0.659$ ). Besides, We further performed univariate and multivariate Cox independent prognostic analysis of the risk score, and the results showed that the risk score is an independent prognostic factor of LUAD. The results of the GESA analysis showed that the poor prognosis of the highrisk group may be related to cell cycle, genetic material metabolism, gene replication, translation process, and other cellular processes and signaling pathways. The results of the GO and KEGG analysis of DEGs between the two groups suggest that $\mathrm{m}^{6} \mathrm{~A}$ modification in LUAD mainly affects the cell cycle, cell senescence, and other biological processes, and thus has an effect on the development and development of LUAD. These results can be used as a theoretical basis for further research on the pathogenesis of LUAD and the establishment of new risk classification and prognostic models.

\section{Conclusions}


In summary, our research shows that $\mathrm{m}^{6} \mathrm{~A}$ RNA methylation regulators are closely related to the occurrence, development, and poor prognosis of LUAD. Three selected $\mathrm{m}^{6} \mathrm{~A}$ RNA methylation regulators (IGF2BP1, HNRNPC, and HNRNPA2B1) were used to construct a risk signature, which was verified to be an independent prognostic factor of LUAD. However, the specific role of IGF2BP1, HNRNPC, and HNRNPA2B1 in LUAD needs further experimental verification.

\section{Abbreviations}

$\mathrm{m}^{6} \mathrm{~A}: \mathrm{N}^{6}$-methyladenosine; LUAD: lung adenocarcinoma; TCGA: The Cancer Genome Atlas; OS: Overall

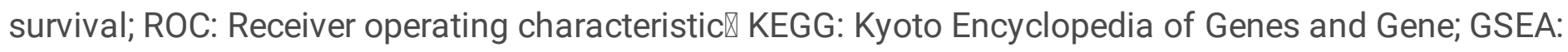
Gene Set Enrichment Analysis; Ontology; GO: Gene ontology; DEGs: the differentially expressed genes.

\section{Declarations}

\section{Acknowledgement}

Not applicable.

\section{Authors' contributions}

SZJ, WR, and FZA conceived and designed this research. WR, FZA, HJ, and HXD collected mRNA transcriptome data and clinical information from the TCGA database. SZJ, FZA, and WR analyzed the data. WR explained the data and wrote the manuscript. SZJ, HJ reviewed and revised the manuscript. All authors read and approved the final manuscript.

\section{Funding}

This work was supported by grants from the Fundamental Research Funds for the Central Universities (WK911000057), the Project of the Science and Technology Innovation of Anhui province (2017070802D146ه2018080402A009), and the Key Programs for Research and Development of Anhui Province (No.1704a0802153).

\section{Availability of data and materials}

Additional data not presented in the manuscript can be obtained by contacting the authors.

\section{Ethics approval and consent to participant}

Not applicable.

\section{Consent for publication}

Not applicable. 


\section{Competing interests}

The authors declare that there are no conflicts of interest.

\section{References}

1. Bray F, Ferlay J, Soerjomataram I, Siegel RL, Torre LA, Jemal A. Global cancer statistics 2018 : GLOBOCAN estimates of incidence and mortality worldwide for 36 cancers in 185 countries. Cancer J Clin. 2018;68(6):394-424.

2. Barta JA, Powell CA, Wisnivesky JP. Global Epidemiology of Lung Cancer. Annals of global health. 2019;85(1).

3. Travis WD, Brambilla E, Noguchi M, Nicholson AG, Geisinger KR, Yatabe Y, et al. International association for the study of lung cancer/american thoracic society/european respiratory society international multidisciplinary classification of lung adenocarcinoma. Journal of thoracic oncology: official publication of the International Association for the Study of Lung Cancer. 2011;6(2):244-85.

4. Siegel RL, Miller KD, Jemal A. Cancer statistics. 2020. CA: a cancer journal for clinicians. 2020;70(1):7-30.

5. Wu K, House L, Liu W, Cho WC. Personalized targeted therapy for lung cancer. Int J Mol Sci. 2012;13(9):11471-96.

6. Wei C, Gershowitz A, Moss B. Methylated nucleotides block 5 ' terminus of HeLa cell messenger RNA. Cell. 1975;4(4):379-86.

7. Jia G, Fu Y, Zhao X, Dai Q, Zheng G, Yang Y, et al. N 6-methyladenosine in nuclear RNA is a major substrate of the obesity-associated FTO. Nature chemical biology. 2011;7(12):885-7.

8. Su R, Dong L, Li C, Nachtergaele S, Wunderlich M, Qing Y, et al. R-2HG exhibits anti-tumor activity by targeting FTO/m6A/MYC/CEBPA signaling. Cell. 2018;172(1-2):90-105. e23.

9. Liu N, Dai Q, Zheng G, He C, Parisien M, Pan T. N 6-methyladenosine-dependent RNA structural switches regulate RNA-protein interactions. Nature. 2015;518(7540):560-4.

10. Geula S, Moshitch-Moshkovitz S, Dominissini D, Mansour AA, Kol N, Salmon-Divon M, et al. m6A mRNA methylation facilitates resolution of naïve pluripotency toward differentiation. Science. 2015;347(6225):1002-6.

11. Sun $T, W u R$, Ming $L$. The role of m6A RNA methylation in cancer. Biomedicine pharmacotherapy $=$ Biomedecine pharmacotherapie. 2019;112:108613.

12. Dominissini D, Moshitch-Moshkovitz S, Schwartz S, Salmon-Divon M, Ungar L, Osenberg S, et al. Topology of the human and mouse m6A RNA methylomes revealed by m6A-sEq. Nature. 2012;485(7397):201-6.

13. Zaccara S, Ries RJ, Jaffrey SR. Reading, writing and erasing mRNA methylation. Nature reviews Molecular cell biology. 2019;20(10):608-24. 
14. Meyer KD, Jaffrey SR. Rethinking m6A Readers, Writers, and Erasers. Annu Rev Cell Dev Biol. 2017;33(1):319-42.

15. Niu Y, Zhao X, Wu YS, Li MM, Wang XJ, Yang YG. N6-methyl-adenosine (m6A) in RNA: an old modification with a novel epigenetic function. Genom Proteom Bioinform. 2013;11(1):8-17.

16. Taketo K, Konno M, Asai A, Koseki J, Toratani M, Satoh T, et al. The epitranscriptome m6A writer METTL3 promotes chemo- and radioresistance in pancreatic cancer cells. Int $\mathrm{J}$ Oncol. 2018;52(2):621-9.

17. Ma Jz, Yang F, Zhou Cc, Liu F, Yuan Jh, Wang F, et al. METTL14 suppresses the metastatic potential of hepatocellular carcinoma by modulating N6-methyladenosine-dependent primary MicroRNA processing. Hepatology. 2017;65(2):529-43.

18. Ritchie ME, Phipson B, Wu D, Hu Y, Law CW, Shi W, et al. limma powers differential expression analyses for RNA-sequencing and microarray studies. Nucleic acids research. 2015;43(7):e47-e.

19. Bøvelstad HM, Nygård S, Størvold HL, Aldrin M, Borgan $\varnothing$, Frigessi A, et al. Predicting survival from microarray data-a comparative study. Bioinformatics. 2007;23(16):2080-7.

20. Sauerbrei W, Royston P, Binder H. Selection of important variables and determination of functional form for continuous predictors in multivariable model building. Statistics in medicine. 2007;26(30):5512-28.

21. Therneau T. A package for survival analysis in S. version 2.38 2015. Available from: https://CRAN.Rproject.org/package=survival.

22. Hanley JA, McNeil BJ. The meaning and use of the area under a receiver operating characteristic (ROC) curve. Radiology. 1982;143(1):29-36.

23. Dolinski K, Dwight S, Eppig J, Harris M, Hill D, Issel-Tarver L, et al. Gene ontology: tool for the unification of biology. the gene ontology consortium. Nat Genet. 2000;25(1):2529Attri.

24. Kanehisa M, Goto S. KEGG: kyoto encyclopedia of genes and genomes. Nucleic acids research. 2000;28(1):27-30.

25. Duma N, Santana-Davila R, Molina JR. Non-Small Cell Lung Cancer: Epidemiology, Screening, Diagnosis, and Treatment. Mayo Clinic proceedings. 2019;94(8):1623-40.

26. Lemjabbar-Alaoui H, Hassan OU, Yang YW, Buchanan P. Lung cancer: Biology and treatment options. Biochim Biophys Acta. 2015;1856(2):189-210.

27. Travis WD, Brambilla E, Riely GJ. New pathologic classification of lung cancer: relevance for clinical practice and clinical trials. J Clin Oncol. 2013;31(8):992-1001.

28. Wang X, Lu Z, Gomez A, Hon GC, Yue Y, Han D, et al. N6-methyladenosine-dependent regulation of messenger RNA stability. Nature. 2014;505(7481):117-20.

29. Liu J, Eckert MA, Harada BT, Liu S-M, Lu Z, Yu K, et al. m 6 A mRNA methylation regulates AKT activity to promote the proliferation and tumorigenicity of endometrial cancer. Nat Cell Biol. 2018;20(9):1074-83. 
30. Lin S, Choe J, Du P, Triboulet R, Gregory RI. The m6A methyltransferase METTL3 promotes translation in human cancer cells. Molecular cell. 2016;62(3):335-45.

31. Guan K, Liu X, Li J, Ding Y, Li J, Cui G, et al. Expression Status And Prognostic Value Of M6Aassociated Genes in Gastric Cancer. J Cancer. 2020;11(10):3027-40.

32. Li J, Zhu L, Shi Y, Liu J, Lin L, Chen X. m6A demethylase FTO promotes hepatocellular carcinoma tumorigenesis via mediating PKM2 demethylation. American journal of translational research. 2019;11(9):6084.

33. Niu Y, Lin Z, Wan A, Chen H, Liang H, Sun L, et al. RNA N6-methyladenosine demethylase FTO promotes breast tumor progression through inhibiting BNIP3. Mol Cancer. 2019;18(1):46.

34. Chen M, Nie ZY, Wen XH, Gao YH, Cao H, Zhang SF. m6A RNA methylation regulators can contribute to malignant progression and impact the prognosis of bladder cancer. Bioscience reports. 2019;39(12).

35. Huang $\mathrm{H}$, Weng $\mathrm{H}$, Sun W, Qin X, Shi H, Wu H, et al. Recognition of RNA N 6-methyladenosine by IGF2BP proteins enhances mRNA stability and translation. Nat Cell Biol. 2018;20(3):285-95.

36. Zhao Y, Shi Y, Shen H, Xie W. m(6)A-binding proteins: the emerging crucial performers in epigenetics. J Hematol Oncol. 2020;13(1):35.

\section{Figures}


A

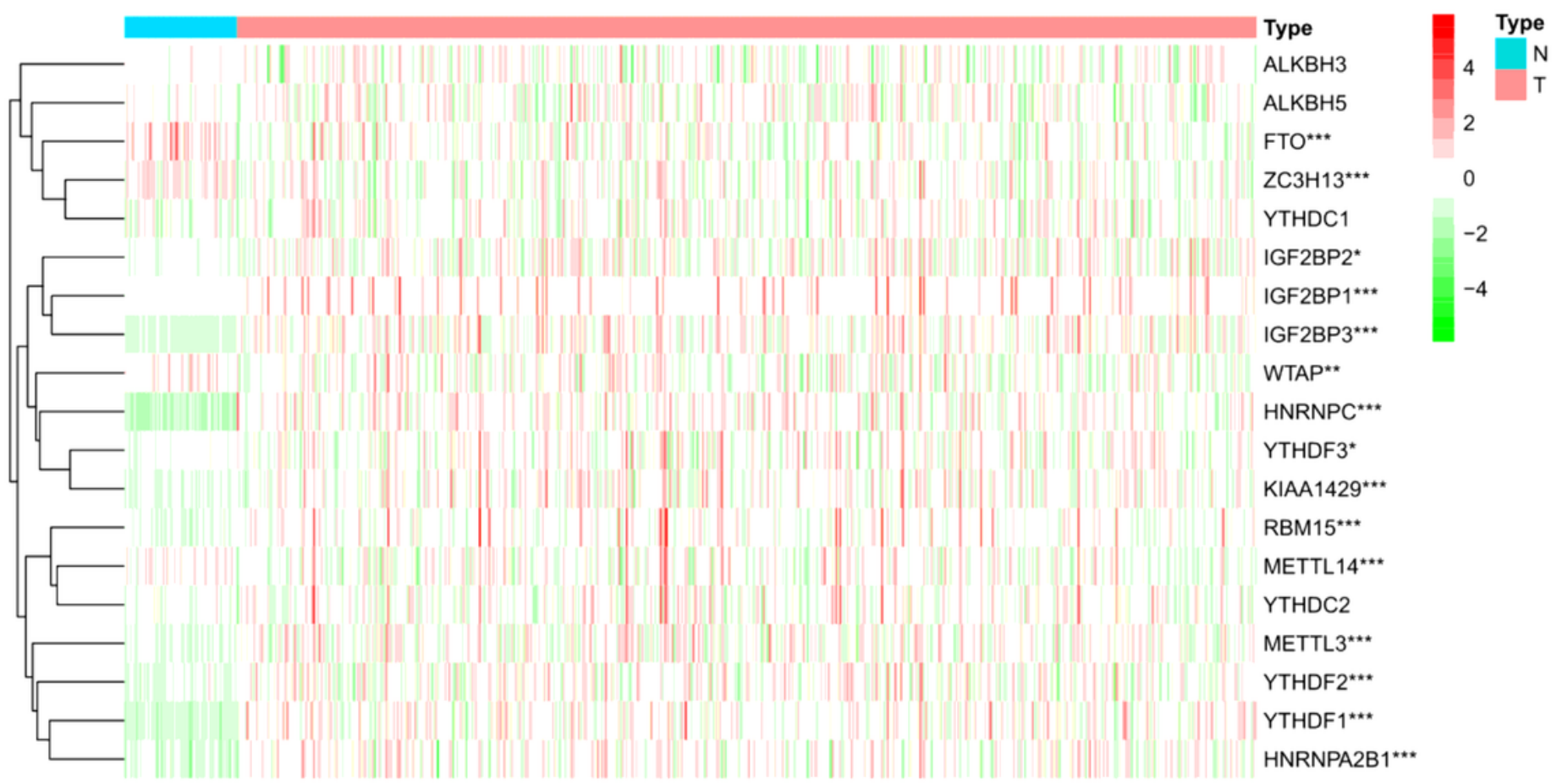

B

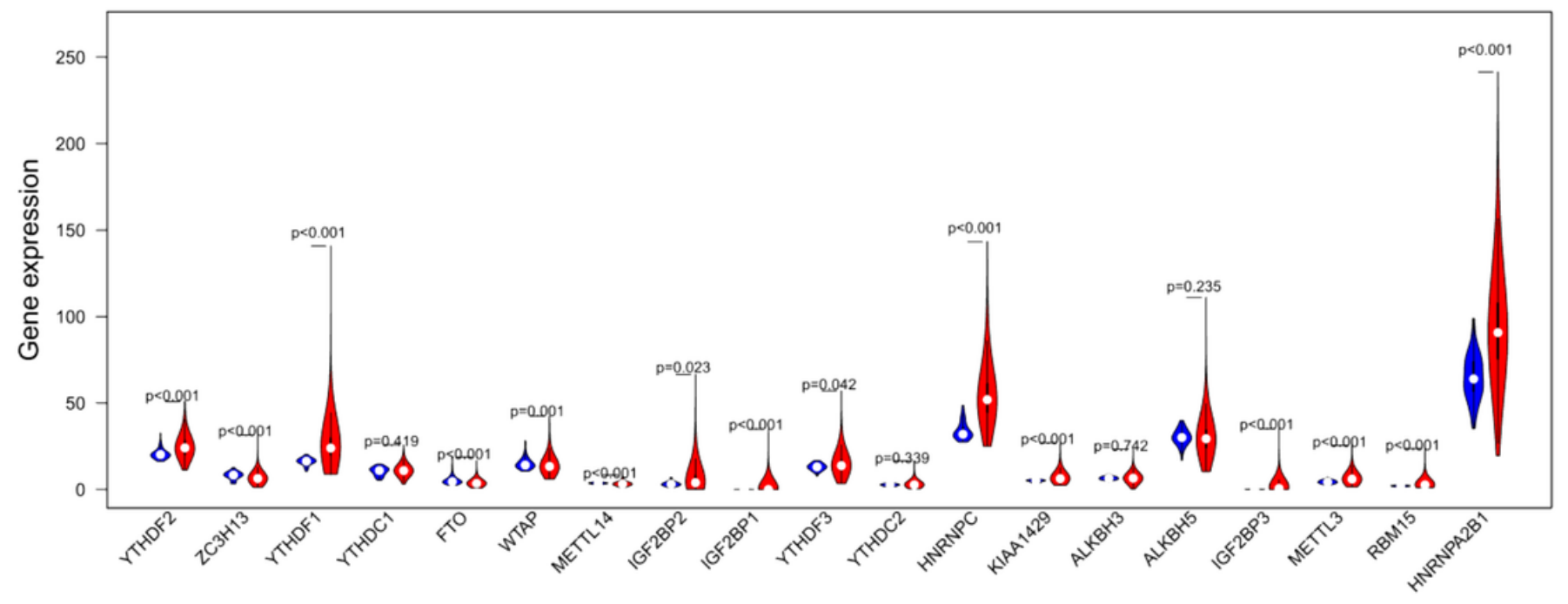

\section{Figure 1}

Bioinformatics analysis of m6A RNA methylation regulator expression in LUAD. (a) Heat map of the expression of m6A RNA methylation regulator in normal tissue ( $N$, blue) and LUAD tumor tissue (T, pink). Red represents up-regulation and green represents down-regulation. (b) Vioplot visualizing the

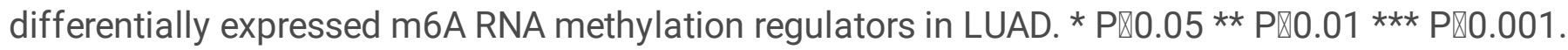




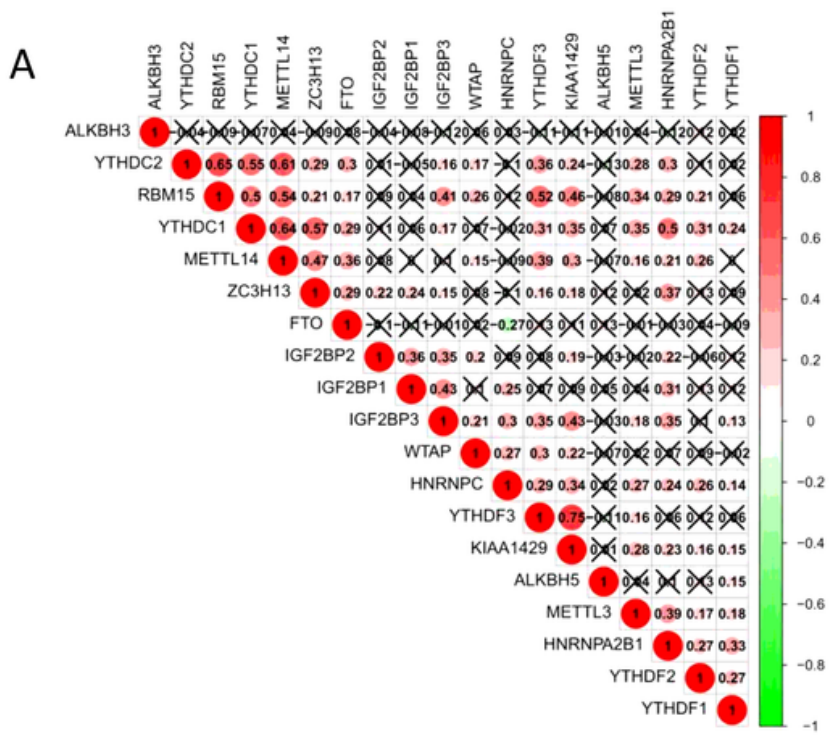

B

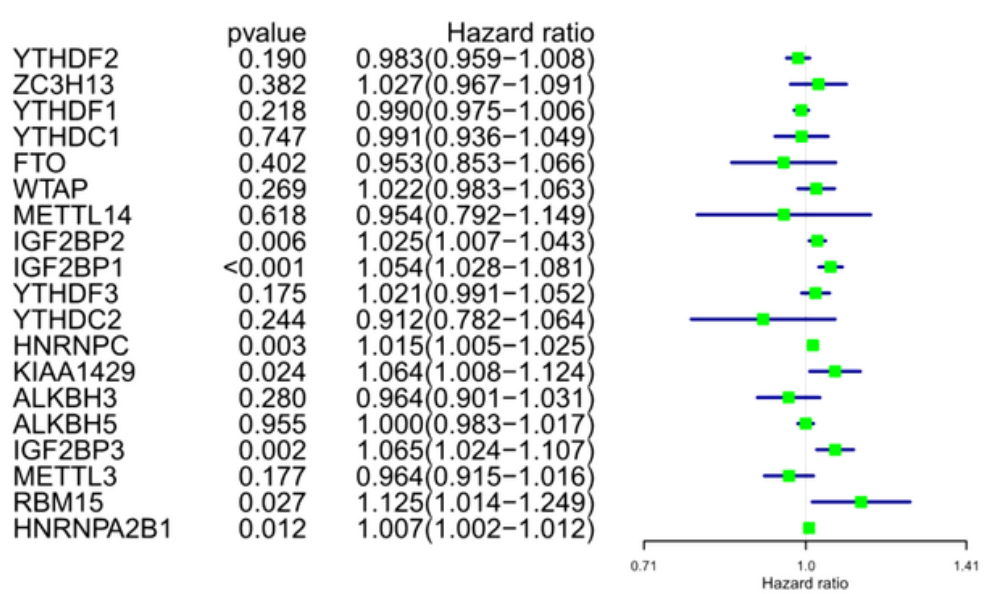

Figure 2

The correlation among 19 selected m6A RNA methylation regulators. (a) Spearman correlation analysis of m6A RNA methylation regulator in LUAD. (b) The hazard ratio (HR) and 95\% confidence interval (Cl) of m6A RNA methylation regulator calculated by univariate COX regression analysis. 
A
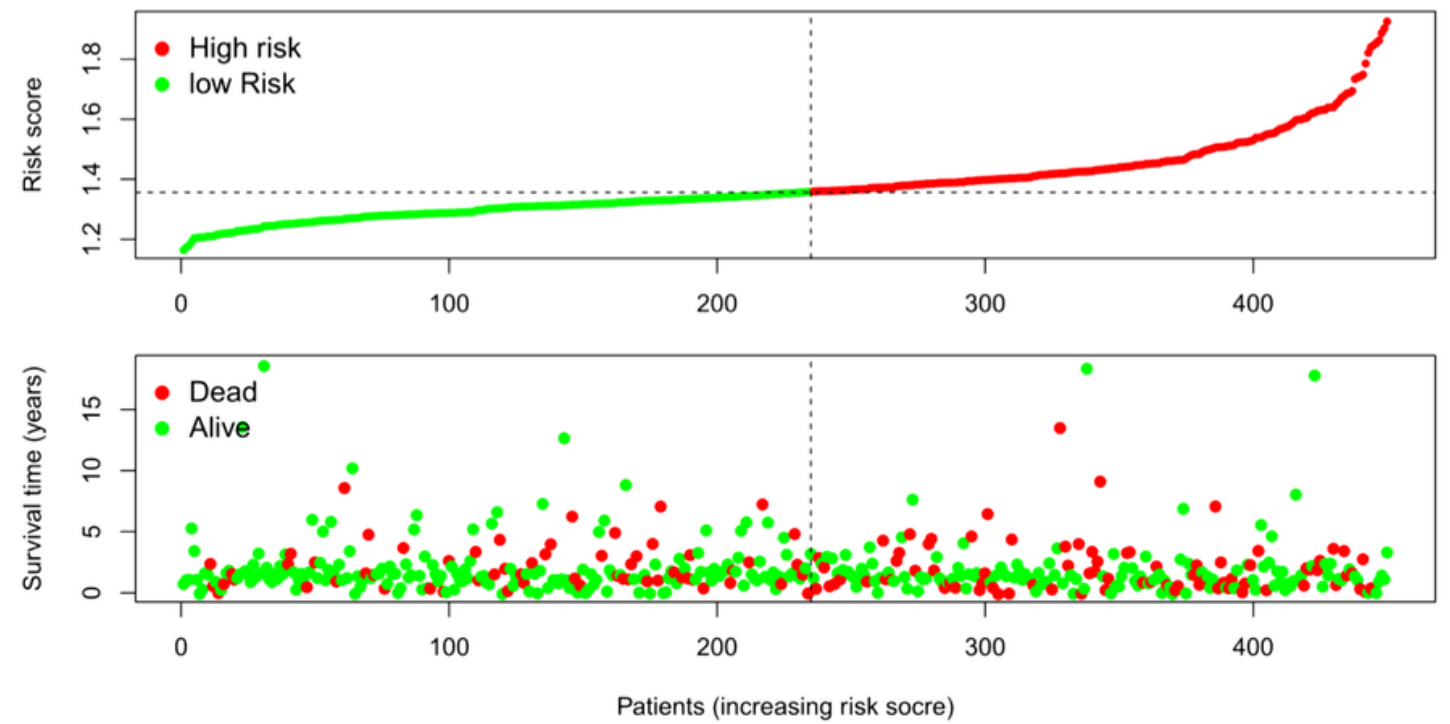

B

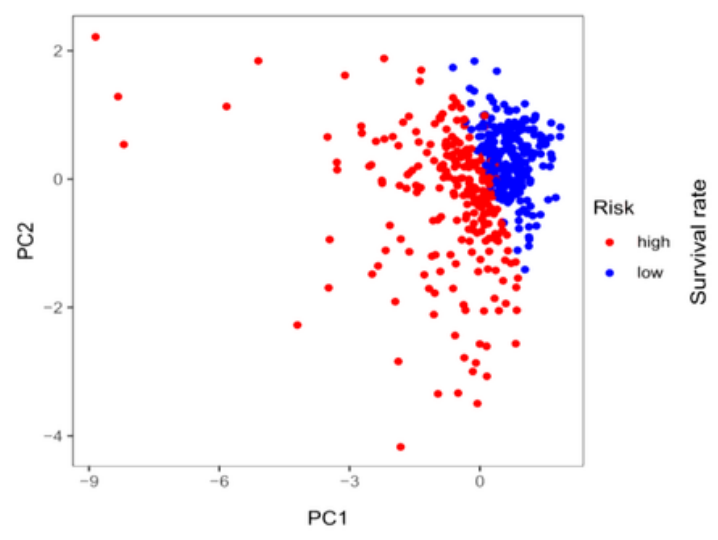

C

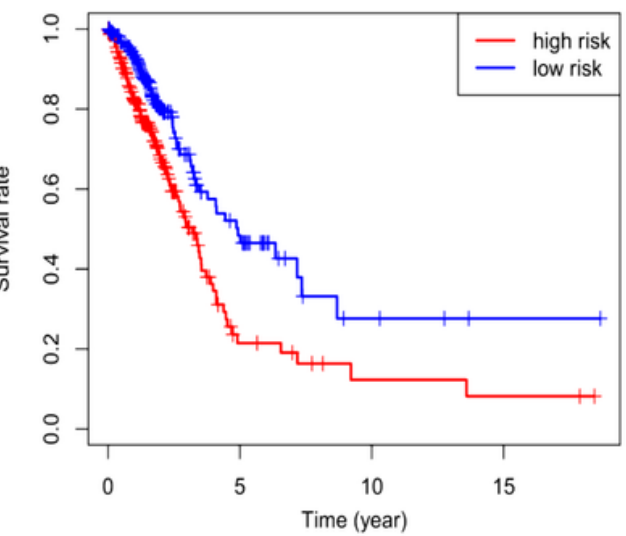

D

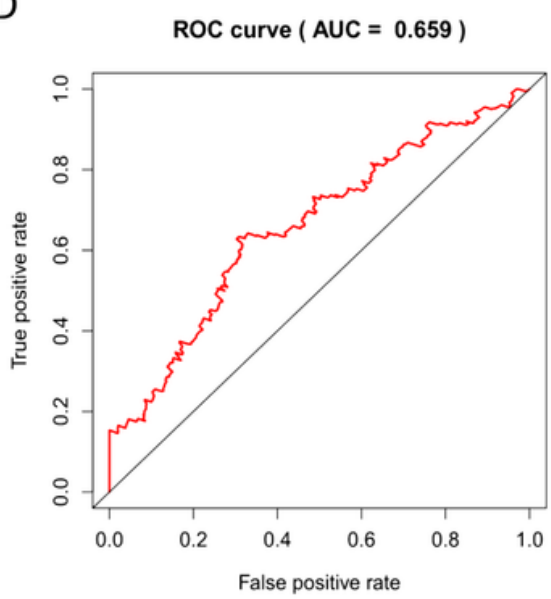

Figure 3

Analysis of survival risk signature for LUAD patients. (a) The risk score curve of each sample in the LUAD data set of the TCGA database. (b) Principal component analysis PCA based on the median grouping of risk scores. (c) Kaplan-Meier survival analysis curve of high-risk and low-risk groups. (d) The constructed ROC curve evaluates the predictive efficiency of the risk signature for the survival rate of LUAD patients, AUC $=0.659$. 


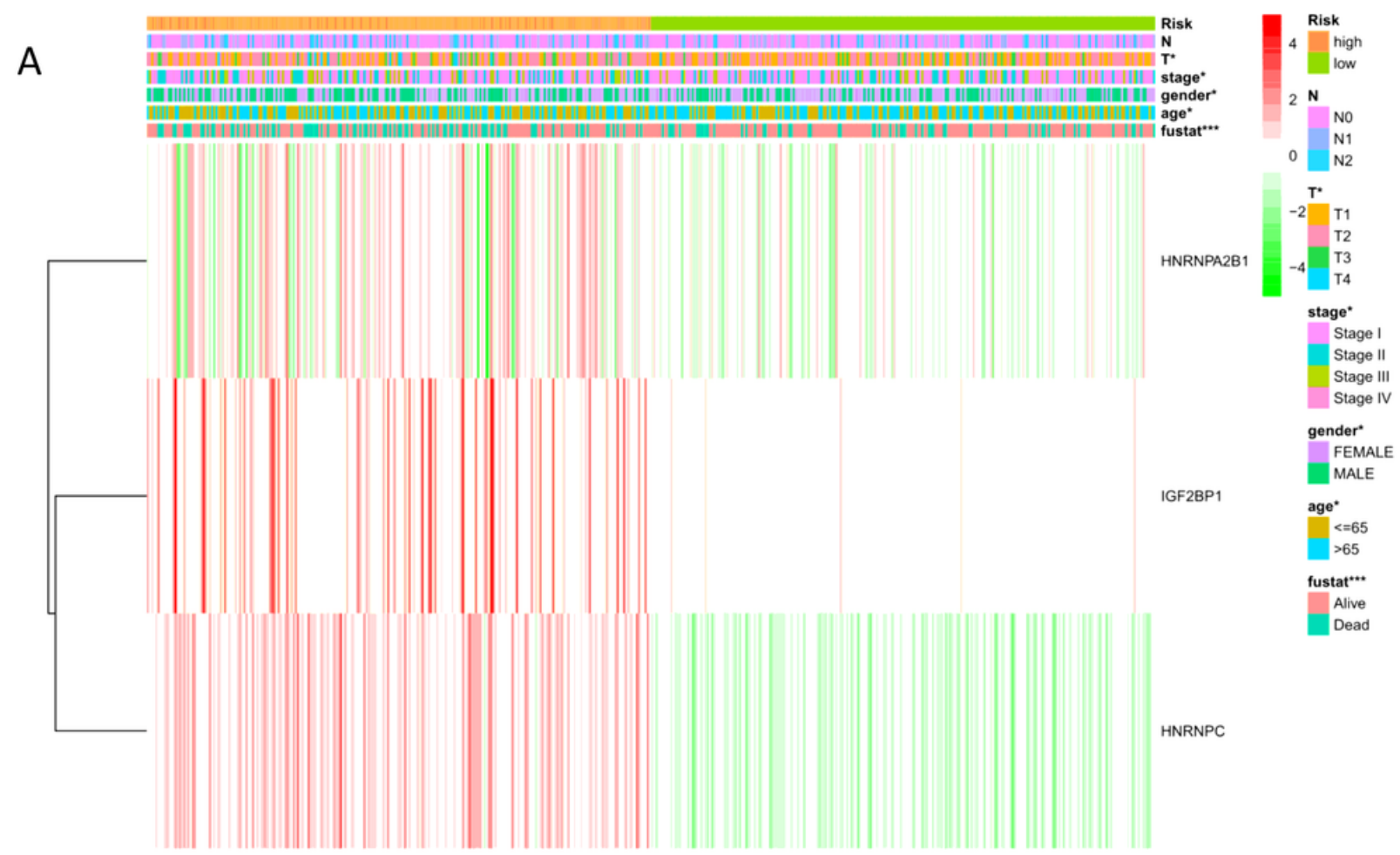

B

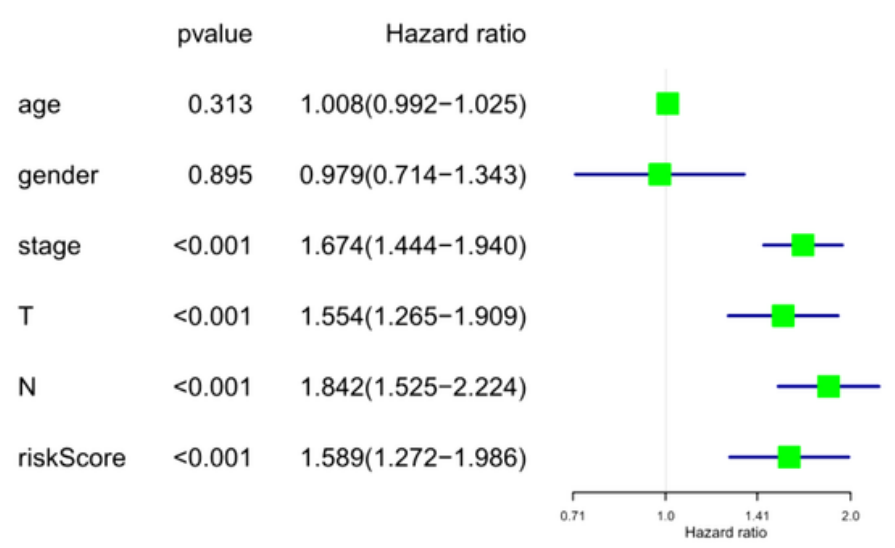

C

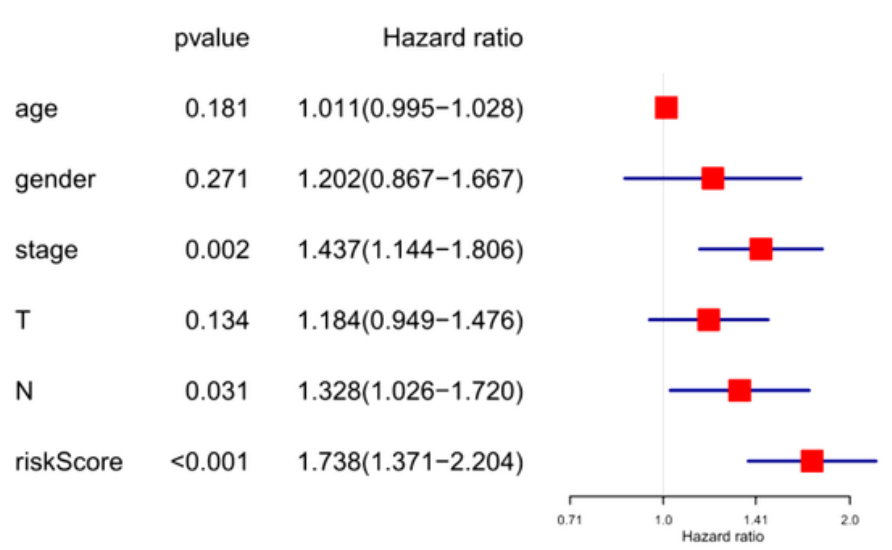

Figure 4

The relationship between risk score and clinicopathological characteristics of LUAD. (a) Heat map of the expression of three m6A RNA methylation regulators and the distribution of clinicopathological variables

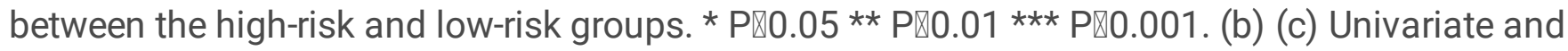
multivariate independent prognostic analysis between clinicopathological characteristics and risk scores and OS of patients. The horizontal line in the color module represents the confidence interval of each factor. 

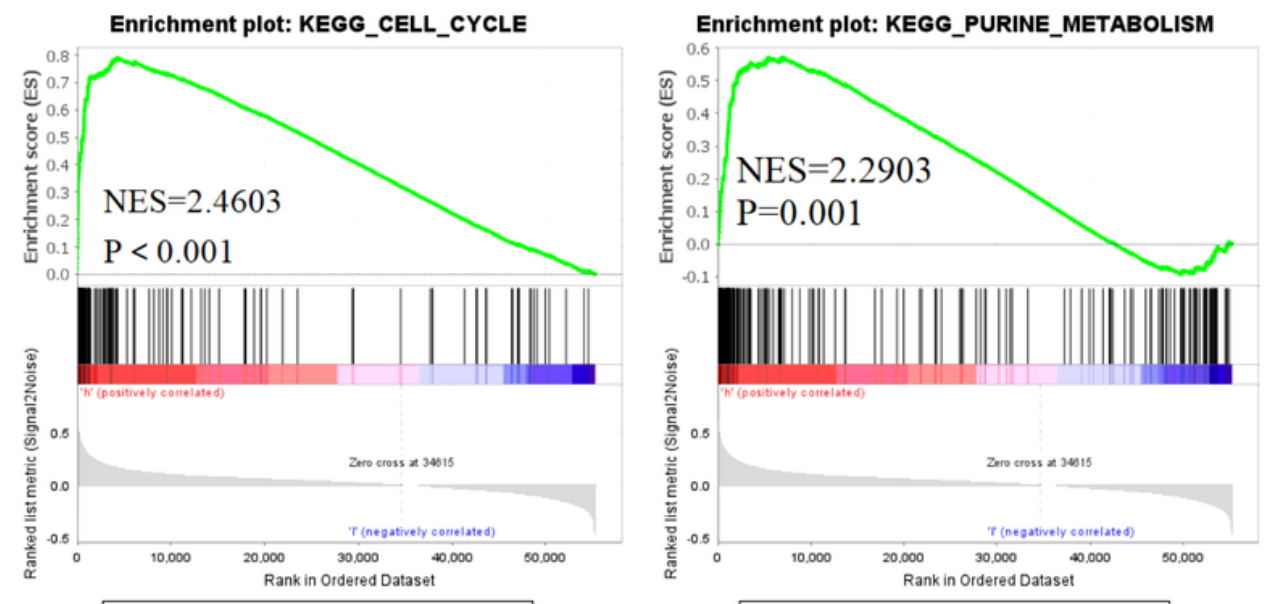

Enrichment plot: KEGG_PYRIMIDINE_METABOLISM
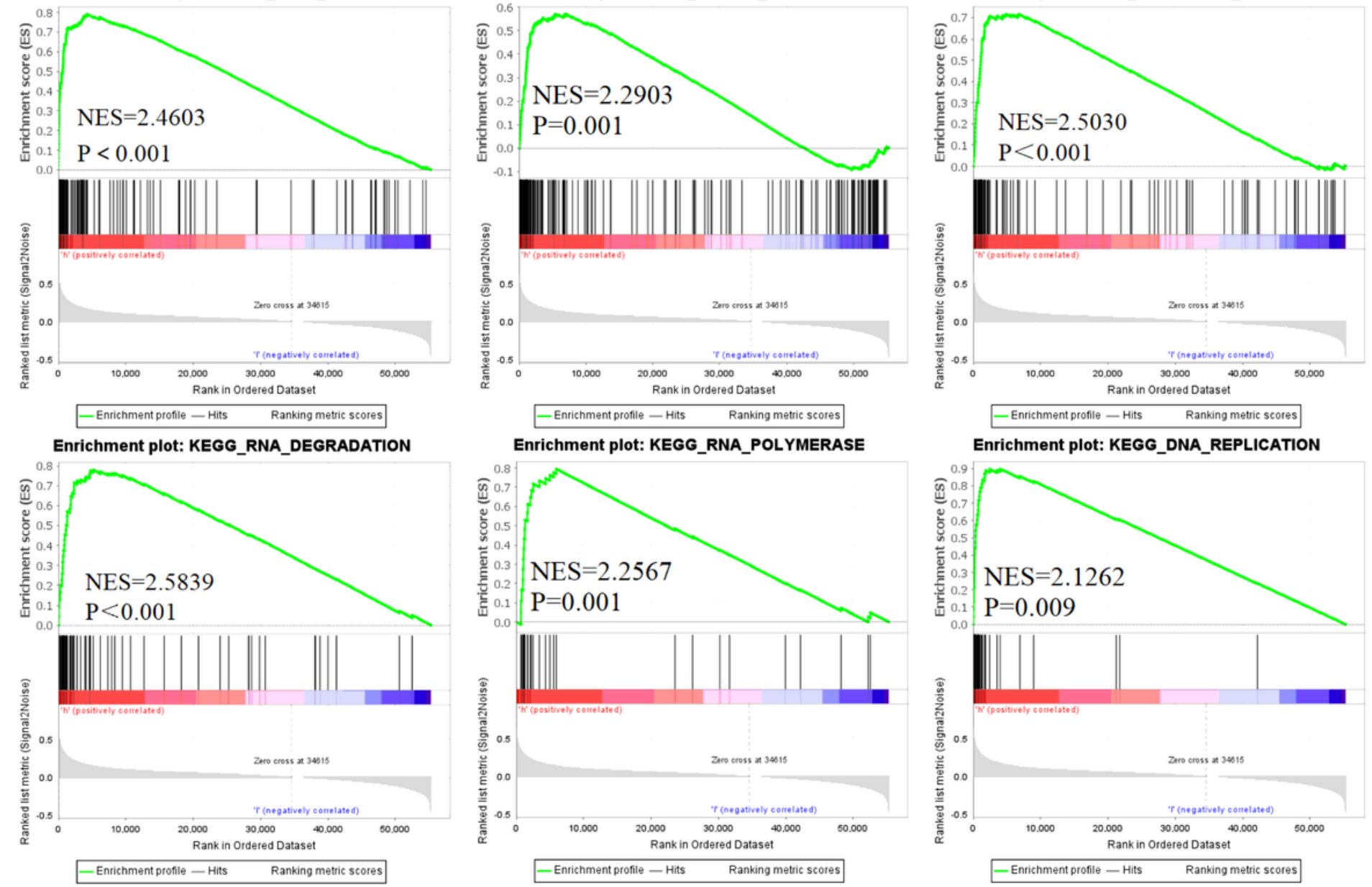

Figure 5

Function enrichment analysis of risk signature based on GSEA. The genes involved in the six functional pathways in the figure were significantly up-regulated in the LUAD high-risk group. 

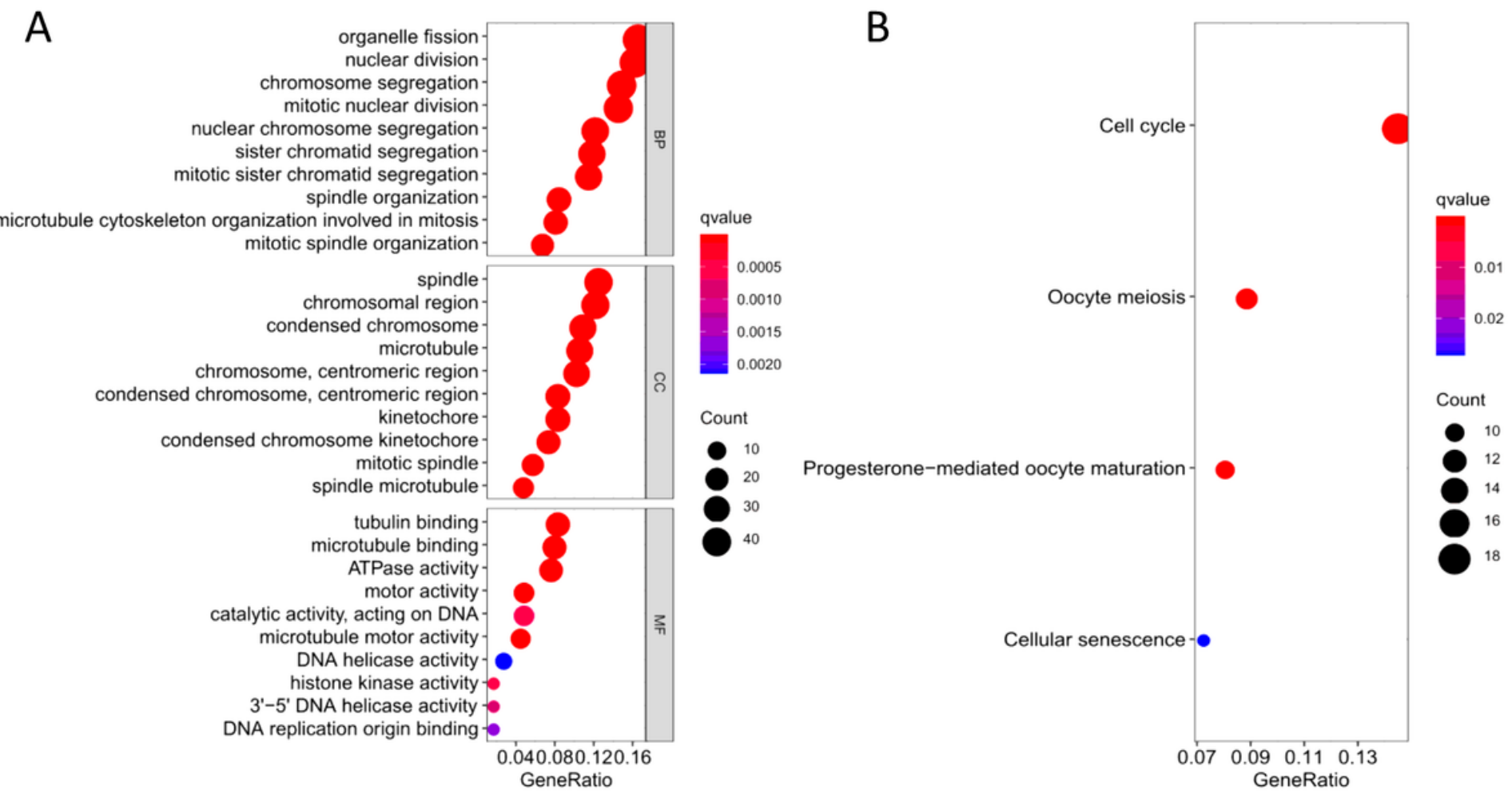

\section{Figure 6}

GO and KEGG analysis of DEGs between high-risk and low-risk groups. (a) (b) The most significant GO enrichment and KEGG pathway in DEGs between the high and low-risk groups. The red depth represents the greater the significance, the blue depth the less the significance, and the bubble size represents the number of genes. 